
\title{
25 Research Sourere \\ Targeting the ARNTL2 Gene as a Potential Strategy for Lung Adenocarcinoma
}

\section{Huan Zhang}

Zhongshan Hospital Fudan University

Ming Li

Zhongshan Hospital Fudan University

\section{Xiangyang Yu}

Chinese Academy of Medical Sciences Cancer Institute and Hospital: Cancer Hospital Chinese Academy of Medical Sciences

\section{Guangyao Shan}

Zhongshan Hospital Fudan University

\section{Xing Jin}

Zhongshan Hospital Fudan University

\section{Mingxiang Feng}

Zhongshan Hospital Fudan University

\section{Cheng Zhan}

Fudan University https://orcid.org/0000-0001-8745-9276

\section{Hao Wang}

Zhongshan Hospital Fudan University

\section{Miao Lin ( $\square$ lin.miao@zs-hospital.sh.cn )}

Zhongshan Hospital Fudan University

\section{Qun Wang}

Zhongshan Hospital Fudan University

\section{Research Article}

Keywords: ARNTL2, lung adenocarcinoma, multi-omics, integrative analysis

Posted Date: November 30th, 2021

DOI: https://doi.org/10.21203/rs.3.rs-1082517/v1

License: (c) (i) This work is licensed under a Creative Commons Attribution 4.0 International License. Read Full License 


\section{Abstract}

The relationship between biorhythm and cancer has been increasingly reported in recent years. As one of the core transcription factors of biorhythm, ARNTL2 is thought to be implicated in the occurrence and development of many malignant tumors, such as breast cancer. However, the role of ARNTL2 in lung adenocarcinoma remains elusive. In the current study, we found that expression of ARNTL2 was markedly upregulated in lung adenocarcinoma, and high ARNTL2 expression was correlated with advanced $\mathrm{N}$ stage and poor survival in patients. Moreover, ARNTL2 expression levels are closely associated with many important features of lung adenocarcinoma, such as tumor immune microenvironment, ferroptosis, microsatellite instability, tumor mutational load, and drug sensitivity. ARNTL2 could also promote proliferation, invasion, and metastasis of lung adenocarcinoma cells. In addition, we found that high ARNTL2 expression might promote epithelial-mesenchymal transition, metastasis and promotion of angiogenesis of tumor cells. In conclusion, our study reveals that ARNTL2 is a prognostic and promising therapeutic biomarker for people with lung adenocarcinoma.

\section{Introduction}

Lung cancer is the foremost cause of cancer death, and it has the second-highest incidence worldwide in $2020^{1}$. Among the primary lung cancers, lung adenocarcinoma is the most common subtype ${ }^{2}$. Although efforts and improvements have been made in therapy for lung adenocarcinoma (LUAD), the prognosis of LUAD patients is still not optimistic, the 5 -year survival rate of lung cancer patients was less than $20 \% 3$. Thus, it is of great importance to determine the etiology and mechanisms of LUAD malignant progression and explore more effective treatment strategies.

Circadian rhythms affect many physiological processes, it is widely disrupted in cancers, and its role in the tumor has also been revealed by increasing studies in recent years. Aryl Hydrocarbon Receptor Nuclear Translocator Like 2 (ARNTL2), as a critical circadian transcription factor ${ }^{4,5}$, has been shown to play an important role in a variety of tumors, such as colorectal cancer, kidney cancer, and pancreatic ductal adenocarcinoma. However, studies on the role of ARNTL2 in lung adenocarcinoma are relatively rare and not sufficiently thorough.

The current study comprehensively explored the relationship between the ARNTL2 and lung adenocarcinoma, in order to explore the roles of ARNTL2 in the development, progression and treatment of lung adenocarcinoma. The effects of ARNTL2 on human lung adenocarcinoma cells in terms of tumor mutational burden (TMB), microsatellite instability (MSI), immune microenvironment, ferroptosis, and drug sensitivity were further evaluated, aiming to provide potential targets and ideas for the treatment of lung adenocarcinoma. In addition, we experimentally demonstrated that ARNTL2 promotes tumor cell proliferation, migration, and invasion in lung adenocarcinoma cell lines A549 and H1299.

\section{Result}




\section{Gene expression with clinical features}

A previous study found that ARNTL2 was frequently highly expressed in many cancers ${ }^{6}$. The differential expression between tumor and adjacent normal tissues for ARNTL2 across TCGA is shown in Figure 1A. To further validate whether ARNTL2 was differentially expressed in lung adenocarcinoma tissues, we conducted an analysis of 513 cases of LUAD patient data from the TCGA database, which showed significantly higher ARNTL2 expression in LUAD tissues than in normal adjacent tissues, $(P<0.001)$. The same conclusion was obtained from the validation after combining the GTEx data (Figure 1B). In addition, we divided the lung adenocarcinoma patients in the TCGA database equally into two groups according to the high and low expression of ARNTL2, and found there were differences in the baseline characteristics of the two groups (Table 1 and Supplementary Figure 1). Specifically, patients in the highARNTL2 group were more male and had more advanced N stage and AJCC stage. 
Table 1

LUAD patient characteristics according to ARNTL2 expression

\begin{tabular}{|c|c|c|c|}
\hline & high- group & low- group & $p$ value \\
\hline Total evaluated & 257 & 256 & \\
\hline Age(years) & & & 0.931 \\
\hline Mean \pm SD & $65.3 \pm 10.3$ & $65.3 \pm 9.8$ & \\
\hline Sex & & & 0.046 \\
\hline male & 130 & 107 & \\
\hline female & 127 & 149 & \\
\hline Smoking history & & & 0.217 \\
\hline No & 34 & 40 & \\
\hline Yes & 213 & 212 & \\
\hline Unknown & 10 & 4 & \\
\hline Pathologic T stage & & & 0.089 \\
\hline $\mathrm{T} 1$ & 72 & 96 & \\
\hline $\mathrm{T} 2$ & 142 & 134 & \\
\hline T3 & 30 & 17 & \\
\hline $\mathrm{T} 4$ & 11 & 8 & \\
\hline Unknown & 2 & 1 & \\
\hline Pathologic $\mathrm{N}$ stage & & & 0.001 \\
\hline NO & 142 & 188 & \\
\hline N1 & 60 & 35 & \\
\hline N2 & 47 & 27 & \\
\hline N3 & 1 & 1 & \\
\hline Unknown & 7 & 5 & \\
\hline Pathologic M stage & & & 0.099 \\
\hline MO & 178 & 166 & \\
\hline M1 & 16 & 9 & \\
\hline Unknown & 63 & 81 & \\
\hline
\end{tabular}




\begin{tabular}{|llll|}
\hline & high- group & low- group & p value \\
\hline Ajcc 8th stage & & & 0.001 \\
\hline 1 & 115 & 159 & \\
\hline 2 & 69 & 52 & \\
\hline 3 & 53 & 31 & \\
4 & 17 & 9 & \\
\hline Unknown & 3 & 5 & 0.781 \\
\hline Radiation & & & \\
\hline Yes & 7 & 6 & 0.350 \\
\hline No/Unknown & 250 & 250 & \\
\hline Chemotherapy & & & \\
\hline Yes & 82 & 72 & \\
\hline No/Unknown & 175 & 184 & \\
\hline
\end{tabular}

\section{Survival analysis}

We analyzed the prognosis of 504 patients with lung adenocarcinoma in the TCGA database in relation to ARNTL2 expression and found that high ARNTL2 expression predicted poor survival. Results are shown in Figure 1C, ARNTL2-high group vs ARNTL2-low group: OS: $\mathrm{HR}=1.53(95 \% \mathrm{Cl}, 1.14-2.05) \mathrm{p}=$ 0.005 ; PFS: $\mathrm{HR}=1.35(95 \% \mathrm{Cl}, 1.03-1.78) \mathrm{p}=0.031$.

For lung adenocarcinoma patients in the TCGA database, we also validated the correlation between survival and clinical factors. A total of 513 lung adenocarcinoma participants were involved in univariate and multivariate analyses to validate survival factors. Univariate analyses revealed that ARNTL2 ( $p$ \ $0.001)$, T stage ( $p \otimes 0.001)$, and $N$ stage ( $p \otimes 0.001)$ were statistically significant predictors of tumorspecific survival. According to multivariate analysis, ARNTL2 ( $p \otimes 0.001)$, T stage $(p=0.005)$, and N stage $(p=0.001)$ remained independent prognostic predictors for LUAD patients. The details of the correlations between survival outcomes and parameters are shown in the following forest plot (Figure 1D).

\section{TMB and mRNAsi analyses}

As one of the immunotherapy biomarkers, tumor mutation burden (TMB) has attracted more and more attention in recent years, we analyzed the relationship between ARNTL2 gene expression and TMB in lung adenocarcinoma patients according to the TCGA database, and the results showed that there was a degree of positive correlation between ARNTL2 gene expression and TMB (Figure 2A), which may 
indicate that in lung adenocarcinoma patients, as ARNTL2 gene expression increases, TMB also increases. Thus ARNTL2 may have a reference value for immunotherapy.

It has been previously shown that mRNAsi is a valid method to evaluate the level of tumor differentiation. Histopathology confirmed that higher values of mRNAsi tend to represent a greater degree of tumor dedifferentiation. AS shown in Figure 2B, in lung adenocarcinoma, the mRNAsi was significantly higher in the lung tumor specimens than in normal lung tissue. Furthermore, compared with the low ARNTL2 expression group, the high ARNTL2 group showed significantly higher mRNAsi, which may be related to the worse prognosis of patients in the high ARNTL2 group.

\section{MSI}

Microsatellites (MS), also known as short tandem repeats (STR) or simple sequence repeats (SSR), are structures consisting of repetitive sequences of 1-6 nucleotides ${ }^{7}$. MSI is an important factor in tumorigenesis and progression and has been extensively studied in tumors such as colorectal cancer. Early diagnosis of MSI is of great significance to the prognosis and treatment of $\mathrm{MSI}^{8}$. We found that the expression level of ARNTL2 and MSI were negatively correlated in lung adenocarcinoma patients (Figure 2C).

\section{Somatic mutations}

Based on previous reports, the number of somatic mutations is thought to correlate with prognosis. The changes in the copy numbers' variation and distribution of somatic mutations in high-ARNTL2 and lowARNTL2 groups were analyzed. The results showed that TP53 had a high mutation rate in the highARNTL2 group ( $55 \%$ vs. $41 \%$, P-value < 0.01$)$. In contrast, some genes, such as RELN $(11 \%$ for the low subgroup and $18 \%$ for the high subgroup), had a lower mutation rate in the high ARNTL2 group (Figure 2D).

\section{DEG, GO, KEGG analyses and single-cell analysis}

The prognosis of lung adenocarcinoma patients with different ARNTL2 expression levels may be related to DEGs. So, we have analyzed the expression profiles from the TCGA data, to derive the landscape of biological differences. The prognosis of lung adenocarcinoma patients with different ARNTL2 expression levels may be related to DEGs. So, we have analyzed the expression of DEGs to derive landscape of biological differences (Figure 3A), and 185 significant different genes were found, including 114 upregulated genes in the high-ARNTL2 group such as SLC2A1, CD109, and ADGRF4 (adjusted P< 0.01), and 71 downregulated genes including C16orf89, IRX5, and IRX3 (Supplementary Table 2).

Functional enrichment of GO and KEGG in the 185 DEGs were analyzed. These pathways were associated with high-ARNTL2 expression. Cell cycle, apoptosis, IL-17 signaling pathway, and p53 signaling pathway ranked top in the high-ARNTL2 group (Figure 3B). 
In addition, we performed a single-cell analysis of lung adenocarcinoma cells through the CancerSEA database. The results showed that ARNTL2 gene expression was highly correlated with Angiogenesis, EMT, and Metastasis (Figure 3C, Supplementary Table 3).

\section{Immune infiltration and immune checkpoint analyses}

The composition and proportion of immune cells in the tumor microenvironment (TME) have a significant impact on tumor development and treatment ${ }^{9}$. In lung adenocarcinoma, CD8+ and CD4+ T cells showed a significant positive correlation with ARNTL2 expression. In contrast, NK cell and Macrophage M2 showed a negative correlation (Figure 4A). In addition, some common genes closely related to immune checkpoints also differed with the high expression of ARNTL2 (Figure 4B). Compared to the low-ARNTL2 expression group, the high expression group had significantly higher expression of CD274, CTLA4, LAG3, and PDCD1, etc. These differences in the immune microenvironment may provide ideas for immunotherapy.

\section{Ferroptosis and drug-sensitive analyses}

Comparison of important genes associated with ferroptosis between the high and low expression ARNTL2 groups showed that ACSL4, CDKN1A, and MT1G, etc. were overexpressed in the high expression group (Figure 4C), which may predict a correlation between ARNTL2 expression and ferroptosis in lung adenocarcinoma.

ANRTL2 expression also appears to have effects on drug sensitivity in lung adenocarcinoma, with IC50s significantly lower in the high ANRTL2 expression group than in the low ANRTL2 expression group for Gefitinib, Gemcitabine, Cisplatin, and Paclitaxel, although there was no significant difference in sensitivity to erlotinib between the two groups (Figure 4D).

\section{IHC and immunofluorescence: ARNTL2 is highly expressed in lung adenocarcinoma tissues}

The results of immunohistochemistry further confirmed the increased expression of ARNTL2 in lung adenocarcinoma. As shown in Figure 5A, the overexpression of ARNTL2 was seen in resected tumor tissues compared with adjacent normal tissues. Similar results were obtained by immunofluorescence analysis (Figure 5B).

\section{ARNTL2 promotes proliferation, invasion, and migration of LUAD cells}

To identify the effect of ARNTL2 on proliferation and migration of lung adenocarcinoma cells, we established cell model of ARNTL2 downregulation by stably transducing with ARNTL2 shRNA-expressing lentiviruses in A549 and H1299 cells. Next, we detected the expression level of ARNTL2 by Western blot after transfection, the stable knockdown efficiency of shRNA at protein expression level was verified (Figure 5C). ARNTL2 knockdown was very efficient in A549 and H1299 cell lines 
We subsequently performed CCK8, Clone formation, wound healing, and transwell assays to explore the effect of ARNTL2 in tumor proliferation and migration. CCK8 assays showed when ARNTL2 was downregulated, compared with the control cells, the proliferation ability was decreased in both A549 and H1299 cells (Figure 5D). To further testify the role of ARNTL2 in lung adenocarcinoma formation, Clone formation of ARNTL2-knockdown and control cells was assessed. The results showed that ARNTL2knockdown cells formed significantly fewer colonies compared to control cells (Figure 5E), which indicates ARNTL2 is essential for the oncogenicity of lung adenocarcinoma cells.

We also investigated whether ARNTL2-knockdown cells affected migratory and invasion. ARNTL2knockdown significantly reduced the migration of A549 and H1299 cells in a wound-healing assay, shControl cells almost recovered the wound within 48 hours, but shARNTL2 cells still have large areas of unhealed wounds (Figure 6A). As shown in figure 6B, ARNTL2-knockdown cells also showed significantly lower infiltration rates in the transwell migration and invasion assays compared to control cells $(p<0.05)$. These results suggest that ARNTL2 may be a key promoter of lung adenocarcinoma cell growth and invasion.

\section{Discussion}

Circadian rhythm disorders may be associated with a variety of diseases, such as obesity and depression ${ }^{10,11}$. In addition, Strong epidemiological evidence links circadian disruption with cancers ${ }^{12}$. Moreover, in recent years, a large number of studies have demonstrated that circadian rhythm disorders may be associated with the development of various cancers in human being ${ }^{13,14}$. As an important biorhythm-related gene, ARNTL has been reported to play an oncogenic role in many human cancers. For example, Prior studies found that upregulation of ARNTL2 is associated with poor survival and immune infiltration in clear cell renal cell carcinoma ${ }^{15}$. Mazzoccoli et al. reported that ARNTL2 is upregulated in colorectal cancer and is related to tumor invasiveness and aggressiveness ${ }^{16}$. There are many other studies that demonstrated that high expression of Arntl2 was associated with poor survival in BRCA, LIHC, and pancreatic ductal adenocarcinoma $6,17,18$.

In this study, we performed a comprehensive analysis by integrating available data from TCGA and GTEx database. We found that ARNTL2 expression was correlated with the pathological N-stage of lung adenocarcinoma patients. Similar results have been reported in colorectal cancer. And patients with high ARNTL2 expression had a worse prognosis and could serve as an independent predictor of prognosis in lung adenocarcinoma. Furthermore, a prognostic nomogram including ARNTL2 based upon the results of multivariate Cox analysis was constructed to predict the long-term survival of LUAD patients. Our study also confirmed that ARNTL2 is upregulated in lung adenocarcinoma, which is consistent with some previous reports: Arntl2 was upregulated in BLCA, BRCA, COAD, and READ ${ }^{18}$. ARNTL2 can also promote proliferation and migration of lung adenocarcinoma cells, which suggests that ARNTL2 may be a key promoter of lung adenocarcinoma cell growth and invasion. In addition, a positive correlation between 
ARNTL2 expression and TMB was found in lung adenocarcinoma. In contrast, MSI showed a negative correlation with ARNTL2 expression.

Although much progress in the treatment of lung cancer has been made in recent decades, lung cancer remains the deadliest malignancy worldwide and significantly affects the quality of life of patients ${ }^{19}$. Especially for advanced patients, the 5-year survival rate of stage IV lung cancer patients is only $4.7 \% 20$. Immunotherapy is currently attracting increasing attention in the treatment of lung cancer. However, there are still many patients who benefit less from immunotherapy due to insensitivity to drugs. Therefore, it is vital to discover new effective prognostic biomarkers and specific immune-related therapeutic targets. Our study shows that in lung adenocarcinoma, ARNTL2 causes a significant impact on the immune microenvironment. For example, ARNTL2 expression levels were strongly positively correlated with CD8+ T cells, conversely, strongly negatively correlated with Natural killer (NK) cells. NK cells play an important role in the induction of both innate and adaptive immune responses. Many studies have demonstrated the critical role of NK cells in the control of lung cancer: NK cells can infiltrate lung cancer and a significant positive correlation between the number of tumor-infiltrating NK cells and the survival rate of patients after surgery was reported ${ }^{21-23}$. Therefore, a previous study suggested that immunotherapy targeting NK cells may be a breakthrough point in treatment for lung cancer ${ }^{24}$. There were also significant differences in immune checkpoints between high-ARNTL2, and low-ARNTL2 group patients. Immunecheckpoint inhibitors (ICls) is currently making a splash in the treatment of non-small cell lung cancer ${ }^{25}$, 26, further research on the relationship between ARNTL2 and immune checkpoints may be meaningful and necessary. In addition, numerous studies have demonstrated that TMB and MSI can be used to predict the effect of immunotherapy ${ }^{27,28}$, for example, tumor cells with high TMB are reported to be more easily recognized by immune cells, and thus respond more significantly to immunotherapy ${ }^{29}$, which may be used in combination with TNM staging to predict the progression and prognosis of patients with advanced tumors. We found that in lung adenocarcinoma, tumor immune infiltration, immune checkpoints, MSI and TMB were all associated with ARNTL2 expression levels. The above findings may deserve more in-depth studies and help with immunotherapy for a subset of lung adenocarcinoma patients in the future.

We found that ARNTL2 gene expression was highly correlated with EMT and Metastasis by single-cell analysis, which This could potentially explain why LUAD patients with high-ARNTL2 expression have a worse prognosis. Indeed, the role of ARNTL2 in promoting tumor invasion and metastasis has been reported in a variety of tumors. A previous study of colorectal cancer found that high ARNTL2 expression was significantly associated with vascular invasion and lymph node metastasis ${ }^{16}$. In addition, several studies have shown that ARNTL2 is associated with the development and metastasis of breast cancer ${ }^{30}$, and $\mathrm{Ha} \mathrm{NH}$, et al. indicated that affect the expression levels of ARNTL2 has a significant effect on metastatic progression in ER-breast cancers ${ }^{31}$. It has also been demonstrated that in PDAC, ARNTL2 could affect tumor proliferation, migration, and invasion through the TGF/BETA pathway ${ }^{6}$. Brady et al. reported that ARNTL2 could drive metastatic self-sufficiency in lung cancer ${ }^{32}$. Moreover, Epithelial-to- 
mesenchymal transition (EMT) has been increasingly recognized to promote carcinoma invasion and metastasis. A previous study confirmed that in colon carcinoma, downregulation of ARNTL2 could suppress tumor cell proliferation and migration via SMOC2-EMT through inactivation of PI3K/AKT signaling pathway ${ }^{33}$. These studies suggest that ARNTL2 may contribute to tumor development through multiple mechanisms, such as the promotion of metastasis and EMT.

By applying bioinformatics analysis, we found that there was a high correlation between ARNTL2 expression level and promotional effect on angiogenesis in lung adenocarcinoma cells. In addition, ARNTL2 expression levels appear to have an effect on ferroptosis and drug sensitivity in lung adenocarcinoma cells. However, there are no reports on related fields, further experimental validation is required. This will be the next step of our future work which may could help to reveal deeper mechanisms.

There were also some limitations to this study. Although this study used a massive cohort of TCGA and GEO databases to develop and validate the role of ARNTL2 in lung adenocarcinoma, selection bias could not be avoided because of the retrospective nature of our study design. In addition, this experiment did not conduct more in-depth experiments to explore the mechanism of ARNTL promoting tumor metastasis and EMT in lung adenocarcinoma, which is also the direction of our future research.

ARNTL2 is highly expressed in lung adenocarcinoma, and high ARNTL2 expression was associated with lymph node metastasis and was an independent predictor of worse prognosis for lung adenocarcinoma patients. Notably, the expression of ARNTL2 in lung adenocarcinoma is associated with a variety of features such as tumor immunity, ferroptosis, MSI, and drug sensitivity, which may provide a prediction and reference for immunotherapy. ARNTL2 may also be associated with the promotion of tumor metastasis, EMT, and angiogenesis. Through experiments, we verified that it could promote the proliferation, invasion and metastasis of lung adenocarcinoma. In conclusion, ARNTL2 may be a potential biomarker for lung adenocarcinoma, it may be useful to predict prognosis and guide the treatment in a subgroup of LUAD patients, and may be a new target for therapeutic approaches.

\section{Methods}

\section{Data processing}

Gene expression data of LUAD patients (FPKM normalized) and corresponding clinical and survival information of The Cancer Genome Atlas (TCGA) were downloaded from the UCSC Xena browser (GDC hub: https://gdc.xenahubs.net) ${ }^{34}$. The data with missing prognosis information, including outcome status and survival time was removed. The edition of lung cancer staging was classified according to the American Joint Committee on Cancer (AJCC) TNM Classification for Lung and Pleural Tumors (eighth edition).

\section{Genome statistical analysis}


TCGA cases were divided into high or low groups based on the median values of ARNTL2 expression level. Genome statistical analyses performed in R version 4.0.3 were as follows:

(a) differentially expressed genes (DEGs): The limma package was used to identify DEGs and miRNAs. The moderated t-test was adopted to calculate DEGs and miRNA expression changes, and the $P$ value was adjusted as FDR by Benjamini and Hochberg method. The log fold change $>0.5$, and the adjusted $P$ value $<0.05^{35}$.

(b) copy number variations (CNV) and microRNAs (miRNAs): the maftools package were used to compare the distribution of somatic mutations and the types of copy number variations ${ }^{36}$. The adjusted $P$ value $<0.01$ was used to assess the significance of the mutational frequency. The somatic mutations and the types of copy number variations between high and low-ARNTL2 groups were compared by Kruskal-Wallis test, and the adjusted $p$-value $<0.05$ were considered statistically significant. Results were shown with the oncoplot function.

(c) GO and KEGG: The involved pathways and biological functions of the DEGs were performed with GO and KEGG pathway enrichment analysis by the "clusterProfiler" R package ${ }^{35}$. The cut-off value was set as adjusted $P<0.05$ and false discovery rate $(F D R)<0.05$.

(d) Ferroptosis analysis: Ferroptosis related genes are derived from Ze-Xian Liu et al.'s systematic analysis of the aberrances and functional implications of Ferroptosis in Cancer ${ }^{37}$.

(e) mRNAi analysis: We use the OCLR algorithm constructed by Malta et al. to calculate mRNAsi ${ }^{38,39}$. Based on the characteristics of mRNA expression, the gene expression profile contains 11,774 genes. We use the same Spearman correlation (RNA expression data), and then subtract the minimum value and divide by the linear transformation of the maximum value maps the dryness index to the range $[0,1]$.

(f) drug sensitivity analysis: We predicted the chemotherapeutic response for each sample based on the largest publicly available pharmacogenomics database [the Genomics of Drug Sensitivity in Cancer (GDSC), https://www.cancerrxgene.org/].The prediction process was implemented by $\mathrm{R}$ package "pRRophetic" where the samples' half-maximal inhibitory concentration (IC50) was estimated by ridge regression and the prediction accuracy. All parameters were set by the default values with removal of the batch effect of combat and tissue type of allSoldTumours, and duplicate gene expression was summarized as mean value ${ }^{40}$.

(g) immune infiltration and immune checkpoints: to make reliable immune infiltration estimations, we utilized the QUANTISEQ method in the R package Immunodeconv ${ }^{41,42}$. SIGLEC15, TIGIT, CD274, HAVCR2, PDCD1, CTLA4, LAG3, and PDCD1LG2 were selected to be immune-checkpoint-relevant transcripts and the expression values of these eight genes were extracted. The QUANTISEQ method (https://quantuseq.stanford.edu) ${ }^{43}$ was selected to analyze the relative levels of the 10 tumor-infiltrating immune cell phenotypes based on the RNA-seq expression profiles. The distribution of 10 tumorinfiltrating immune cell types between worse and better prognostic signature was investigated. 
(h) Correlation analysis of ARNTL2 expression and TMB/MSI ${ }^{44,45}$ : we used Spearman's correlation analysis to describe the correlation between quantitative variables without a normal distribution. The value range of Pearson correlation coefficient is $[-1,1]$ with a higher absolute value indicating a stronger association and the sign indicating a positive or negative association between the two variables. The density curve on the right represents the distribution trend of the TMB/MSI score; the upper density curve represents the distribution trend of the gene.

All the above analysis methods and $\mathrm{R}$ package were implemented by $\mathrm{R}$ foundation for statistical computing (2020) version 4.0.3.

\section{Single-cell analysis}

Single-cell analysis was performed through the CANCERSEA website. CancerSEA is the first dedicated database that aims to comprehensively decode distinct functional states of cancer cells at single-cell resolution ${ }^{46}$.

\section{Prognosis analysis}

Prognostic information was analyzed in the PrognoScan database and Kaplan-Meier plotter database ${ }^{47}$.

\section{Statistical analysis}

Statistical analyses in the current study were completed by R version 3.6.1 (R Foundation for Statistical Computing, Vienna, Austria). The R package included survival, rms and ggplot2. Statistical significance was set at a two-sided $p$ value $<0.05$. Categorical variables were compared using Fisher's exact test and Pearson's c2 test and continuous variables were compared using Student's t-test and the Wilcoxon test. Multivariable Cox regression analyses were used to test independent prognostic value using the $\mathrm{R}$ package survival and the coxph function.

\section{Cell culture and lentivirus infection}

Lung adenocarcinoma cell lines (A549 and H1299) were purchased from the Chinese Academy of Sciences Cell Bank. Cells were cultured in DMEM (Hyclone, Logan, UT, USA) supplemented with $10 \%$ fetal bovine serum (Hyclone), $100 \mathrm{U} / \mathrm{mL}$ penicillin, and $100 \mathrm{U} / \mathrm{mL}$ streptomycin in a humidified $5 \% \mathrm{CO} 2$ atmosphere at $37^{\circ} \mathrm{C}$.

Two different short hairpin RNAs (shRNAs) encoding shARNTL2 and scramble shRNA control (catalogue) were designed by Shanghai Genechem Co.,Ltd. and cloned into a lentivirus vector with puromycin resistance, which was then transfected into cells and screened with puromycin to ensure transfection efficacy. Viral transduction was performed as the manufacturer's protocol. After 3 days of virus transfection, knockdown was verified by western blot analysis using ARNTL2-specific antibody (ab221557, abcam). Sequences of the shRNAs and the control are provided in Supplementary table 1.

\section{Western blot}


RIPA buffer (Beyotime, Shanghai, China) containing protease and phosphatase inhibitors cocktail (Beyotime) were used to extract proteins, enhanced BCA Protein Assay Kit (Beyotime) was used for protein quantification. As previously reported, Protein samples were separated by electrophoresis on SDSPAGE and transferred to a polyvinylidene difluoride membrane (Merck-Millipore, Burlington, MA, USA). After the transfer, the blots were blocked with $5 \%$ milk for 1 hour and incubated with primary antibody for $12 \mathrm{~h}$ at $4^{\circ} \mathrm{C}$. Then, tris-buffered saline Tween-20 (TBST) was used to wash the membranes three times. After washing, the membranes were incubated with secondary antibodies at room temperature for $1 \mathrm{~h}$. Finally, the protein bands were visualized and analyzed using a Moon Chemiluminescence Reagent kit (Beyotime). The following antibodies were used in this study: Anti-ANRTL2 antibody (1:1,000, abcam, ab221557)

\section{Cell proliferation assay}

We seeded 2000 cells that were in the logarithmic growth phase, per well in a final volume of $100 \mu \mathrm{L}$ growth medium into black 96 -well plates. After incubation at $37^{\circ} \mathrm{C}$ for $0,24,48,72,96$, and $120 \mathrm{~h}$, the cell proliferation was measured by Enhanced Cell Counting Kit-8 Viability Assay Kit (Beyotime).

\section{Wound healing assay}

Control shRNA and shARNTL2 cells were inoculated on the 6-well plate on average. Cells were grown into monolayer and manual scratching with a $200-\mu$ pipette tip, then cells were rinsed with PBS and incubated at $37^{\circ} \mathrm{C}$ in serum-free media. Photographs of the wounded areas were taken every $24 \mathrm{~h}$ by phase-contrast microscopy.

\section{Transwell migration and invasion assay}

Cell migration and invasion abilities were measured by Transwell assays using the 24-well transwell chambers with $8 \mu \mathrm{m}$ polycarbonate membranes (Corning, NY). the uncoated were used to determine migration and pre-coated with Matrigel Basement Membrane Matrix were used to determine invasion (BD Biosciences). The chambers were rehydrated in a serum-free medium for 2 hours as described by the manufacturer. Then the upper chambers were added with serum-free medium, while the lower chambers were added with serum medium. Cells were seeded onto the upper chambers with a density of $5 \times 104$ cells per well, and incubated for $24 \mathrm{~h}$ at $37^{\circ} \mathrm{C}, 5 \% \mathrm{CO}$. Cells migrated toward the lower chambers were fixed with methanol and stained with $0.5 \%$ crystal violet. Each assay was photographed under the inverted microscope (Olympus), and the number of cells within each chamber was counted by ImageJ software.

\section{Clone formation assay}

Control or ARNTL2 shRNA-transduced A549 and H1299 cells ( $3 \times 103$ cells/well) were cultured in 6-well plates were cultured at $37^{\circ} \mathrm{C}$ in $5 \% \mathrm{CO} 2$ environment. ARNTL2 shRNA-transduced or control cells were seeded in 6-well plates at a density of $5 \times 106$ cells per well and cultured at $37^{\circ} \mathrm{C}$ in $5 \% \mathrm{CO} 2$ environment. After 9 days, cells were stained with $4 \%$ formaldehyde $/ 0.005 \%$ gentian violet solution and captured under the inverted microscope. 


\section{Immunohistochemistry and immunofluorescence}

The tissue specimens were collected from both tumor and adjacent normal tissues of 100 patients with LUAD who received surgery from September to November 2020 in the Zhongshan Hospital. As previously reported ${ }^{48}$, the tissues were stained by a GTVision + Detection System/Mo\&Rb Immunohistochemistry kit (GK500710, GeneTech, Shanghai, China) following the manufacturer's protocol. Specifically, the 5- $\mu \mathrm{m}$ paraffin-embedded tissues were dewaxed, rehydrated, and incubated with antibodies against ARNTL2 (1:500, Abcam, Cambridge, UK) at $4^{\circ} \mathrm{C}$ overnight, and then were incubated with biotinylated secondary antibodies. For immunofluorescence, sections were incubated with primary antibodies against ARNTL2 (rabbit polyclonal, 1:500), followed by incubation with the respective secondary antibodies (Cy3-labeled goat anti-rabbit lgG). DAPI nuclear counterstaining was then performed. Finally, fluorescence microscope was used to take micrographs.

\section{Abbreviations}

LUAD: lung adenocarcinoma

ARNTL2: Aryl Hydrocarbon Receptor Nuclear Translocator Like 2

TMB: tumor mutation burden

MSI: Microsatellite instability

TCGA: The Cancer Genome Atlas

AJCC: American Joint Committee on Cancer

DEGs: differentially expressed genes

CNV: copy number variations

miRNAs: microRNAs

GO: Gene Ontology

KEGG: Kyoto Encyclopedia of Genes and Genomes

FDR: false discovery rate

GDSC: the Genomics of Drug Sensitivity in Cancer

IC50: half-maximal inhibitory concentration

shRNAs: short hairpin RNAs 
qRT-PCR: Quantitative real-time polymerase chain reaction

TBST: tris-buffered saline Tween-20

MS: Microsatellites

STR: short tandem repeats

SSR: simple sequence repeats

TME: tumor microenvironment

NK cells: Natural killer cells.

ICls: immune-checkpoint inhibitors

EMT: Epithelial-to-mesenchymal transition

\section{Declarations}

\section{Acknowledgments}

The authors appreciate the academic support from the Home for Researchers. We also have asked the International Science Editing Corporation to edit the language.

\section{Formatting of funding sources}

This work was supported by the Shanghai Medical Innovation Research Project (Grant No. 20Y11908200).

\section{Author Contributions}

Conceptualization was contributed by $\mathrm{HW}, \mathrm{ML}$, and QW; Data collection and curation were contributed by $H Z$, GS, and XJ; Data analysis and interpretation were contributed by ML, HZ, GS, XJ, MF, HW, and ZC; Draft of the manuscript was contributed by $\mathrm{HZ}, \mathrm{ML}$ and $\mathrm{XY}$; Critical revision of the manuscript was contributed by MF, and CZ; Final approval of manuscript and submission were contributed by all authors.

\section{Ethics approval and consent to participate}

Not applicable.

\section{Consent for publication}

Not applicable

\section{Availability of data and materials}


The datasets used and/or analyzed during the current study are available from the corresponding author on reasonable request.

\section{Competing interests}

There are no conflicts of interest to declare.

\section{Disclosures}

The authors declare that there is no conflict of interest.

\section{References}

1. Sung H, Ferlay J, Siegel RL, et al. Global Cancer Statistics 2020: GLOBOCAN Estimates of Incidence and Mortality Worldwide for 36 Cancers in 185 Countries. CA Cancer J Clin. May 2021;71(3):209-249. doi:10.3322/caac. 21660

2. Travis WD, Brambilla E, Noguchi $M$, et al. International association for the study of lung cancer/american thoracic society/european respiratory society international multidisciplinary classification of lung adenocarcinoma. J Thorac Oncol. Feb 2011;6(2):244-85. doi:10.1097/JTO.0b013e318206a221

3. Allemani C, Matsuda T, Di Carlo V, et al. Global surveillance of trends in cancer survival 2000-14 (CONCORD-3): analysis of individual records for 37513025 patients diagnosed with one of 18 cancers from 322 population-based registries in 71 countries. Lancet. Mar 17 2018;391(10125):1023-1075. doi:10.1016/s0140-6736(17)33326-3

4. Nader N, Chrousos GP, Kino T. Circadian rhythm transcription factor CLOCK regulates the transcriptional activity of the glucocorticoid receptor by acetylating its hinge region lysine cluster: potential physiological implications. Faseb j. May 2009;23(5):1572-83. doi:10.1096/fj.08-117697

5. Marti AR, Patil S, Mrdalj J, et al. No Escaping the Rat Race: Simulated Night Shift Work Alters the Time-of-Day Variation in BMAL1 Translational Activity in the Prefrontal Cortex. Front Neural Circuits. 2017;11:70. doi:10.3389/fncir.2017.00070

6. Wang Z, Liu T, Xue W, et al. ARNTL2 promotes pancreatic ductal adenocarcinoma progression through TGF/BETA pathway and is regulated by miR-26a-5p. Cell Death Dis. Aug 10 2020;11(8):692. doi:10.1038/s41419-020-02839-6

7. Garrido-Ramos MA. Satellite DNA: An Evolving Topic. Genes (Basel). Sep 18 2017;8(9)doi:10.3390/genes8090230

8. Li K, Luo H, Huang L, Luo H, Zhu X. Microsatellite instability: a review of what the oncologist should know. Cancer Cell Int. 2020;20:16. doi:10.1186/s12935-019-1091-8

9. Bi G, Chen Z, Yang X, et al. Identification and validation of tumor environment phenotypes in lung adenocarcinoma by integrative genome-scale analysis. Cancer Immunol Immunother. Jul 2020;69(7):1293-1305. doi:10.1007/s00262-020-02546-3 
10. Albrecht U. The circadian clock, metabolism and obesity. Obes Rev. Feb 2017;18 Suppl 1:25-33. doi:10.1111/obr.12502

11. Pinho M, Sehmbi M, Cudney LE, et al. The association between biological rhythms, depression, and functioning in bipolar disorder: a large multi-center study. Acta Psychiatr Scand. Feb 2016;133(2):102-108. doi:10.1111/acps.12442

12. Kakizaki M, Inoue K, Kuriyama S, et al. Sleep duration and the risk of prostate cancer: the Ohsaki Cohort Study. Br J Cancer. Jul 8 2008;99(1):176-8. doi:10.1038/sj.bjc.6604425

13. Kelleher FC, Rao A, Maguire A. Circadian molecular clocks and cancer. Cancer Lett. Jan 1 2014;342(1):9-18. doi:10.1016/j.canlet.2013.09.040

14. Gery S, Koeffler HP. Circadian rhythms and cancer. Cell Cycle. Mar 15 2010;9(6):1097-103. doi:10.4161/cc.9.6.11046

15. Wang S, Ma X, Ying Y, et al. Upregulation of ARNTL2 is associated with poor survival and immune infiltration in clear cell renal cell carcinoma. Cancer Cell Int. Jul 3 2021;21(1):341. doi:10.1186/s12935-021-02046-z

16. Mazzoccoli G, Pazienza V, Panza A, et al. ARNTL2 and SERPINE1: potential biomarkers for tumor aggressiveness in colorectal cancer. J Cancer Res Clin Oncol. Mar 2012;138(3):501-11. doi:10.1007/s00432-011-1126-6

17. Cadenas $C$, van de Sandt L, Edlund K, et al. Loss of circadian clock gene expression is associated with tumor progression in breast cancer. Cell Cycle. 2014;13(20):3282-91. doi:10.4161/15384101.2014.954454

18. Barbosa Vieira TK, Jurema da Rocha Leão M, Pereira LX, et al. Correlation between circadian rhythm related genes, type 2 diabetes, and cancer: Insights from metanalysis of transcriptomics data. Mol Cell Endocrinol. Apr 15 2021;526:111214. doi:10.1016/j.mce.2021.111214

19. Siddiqui F, Siddiqui AH. Lung Cancer. StatPearls. StatPearls Publishing Copyright $@$ 2021, StatPearls Publishing LLC.; 2021.

20. Fehlmann T, Kahraman M, Ludwig N, et al. Evaluating the Use of Circulating MicroRNA Profiles for Lung Cancer Detection in Symptomatic Patients. JAMA Oncol. May 1 2020;6(5):714-723. doi:10.1001/jamaoncol.2020.0001

21. Villegas FR, Coca S, Villarrubia VG, et al. Prognostic significance of tumor infiltrating natural killer cells subset CD57 in patients with squamous cell lung cancer. Lung Cancer. Jan 2002;35(1):23-8. doi:10.1016/s0169-5002(01)00292-6

22. Takanami I, Takeuchi K, Giga M. The prognostic value of natural killer cell infiltration in resected pulmonary adenocarcinoma. J Thorac Cardiovasc Surg. Jun 2001;121(6):1058-63. doi:10.1067/mtc.2001.113026

23. Jin S, Deng Y, Hao JW, et al. NK cell phenotypic modulation in lung cancer environment. PLoS One. 2014;9(10):e109976. doi:10.1371/journal.pone.0109976

24. Zeng Y, Lv X, Du J. Natural killer cell-based immunotherapy for lung cancer: Challenges and perspectives (Review). Oncol Rep. Nov 2021;46(5)doi:10.3892/or.2021.8183 
25. Zhou F, Qiao M, Zhou C. The cutting-edge progress of immune-checkpoint blockade in lung cancer. Cell Mol Immunol. Feb 2021;18(2):279-293. doi:10.1038/s41423-020-00577-5

26. Guo H, Li W, Qian L, Cui J. Clinical challenges in neoadjuvant immunotherapy for non-small cell lung cancer. Chin J Cancer Res. Apr 30 2021;33(2):203-215. doi:10.21147/j.issn.1000-9604.2021.02.08

27. Teng MW, Ngiow SF, Ribas A, Smyth MJ. Classifying Cancers Based on T-cell Infiltration and PD-L1. Cancer Res. Jun 1 2015;75(11):2139-45. doi:10.1158/0008-5472.Can-15-0255

28. Yarchoan M, Hopkins A, Jaffee EM. Tumor Mutational Burden and Response Rate to PD-1 Inhibition. N Engl J Med. Dec 21 2017;377(25):2500-2501. doi:10.1056/NEJMc1713444

29. Singal G, Miller PG, Agarwala V, et al. Association of Patient Characteristics and Tumor Genomics With Clinical Outcomes Among Patients With Non-Small Cell Lung Cancer Using a Clinicogenomic Database. Jama. Apr 9 2019;321(14):1391-1399. doi:10.1001/jama.2019.3241

30. Mocellin S, Tropea S, Benna C, Rossi CR. Circadian pathway genetic variation and cancer risk: evidence from genome-wide association studies. BMC Med. Feb 19 2018;16(1):20. doi:10.1186/s12916-018-1010-1

31. Ha NH, Long J, Cai Q, Shu XO, Hunter KW. The Circadian Rhythm Gene Arntl2 Is a Metastasis Susceptibility Gene for Estrogen Receptor-Negative Breast Cancer. PLoS Genet. Sep 2016;12(9):e1006267. doi:10.1371/journal.pgen.1006267

32. Brady JJ, Chuang CH, Greenside PG, et al. An Arntl2-Driven Secretome Enables Lung Adenocarcinoma Metastatic Self-Sufficiency. Cancer Cell. May 9 2016;29(5):697-710. doi:10.1016/j.ccell.2016.03.003

33. Lu M, Huang L, Tang $Y$, et al. ARNTL2 knockdown suppressed the invasion and migration of colon carcinoma: decreased SMOC2-EMT expression through inactivation of PI3K/AKT pathway. Am J Transl Res. 2020;12(4):1293-1308.

34. Tang Z, Li C, Kang B, Gao G, Li C, Zhang Z. GEPIA: a web server for cancer and normal gene expression profiling and interactive analyses. Nucleic Acids Res. Jul 3 2017;45(W1):W98-w102. doi:10.1093/nar/gkx247

35. Yu G, Wang LG, Han Y, He QY. clusterProfiler: an R package for comparing biological themes among gene clusters. Omics. May 2012;16(5):284-7. doi:10.1089/omi.2011.0118

36. Mayakonda A, Lin DC, Assenov Y, Plass C, Koeffler HP. Maftools: efficient and comprehensive analysis of somatic variants in cancer. Genome Res. Nov 2018;28(11):1747-1756. doi:10.1101/gr.239244.118

37. Liu Z, Zhao Q, Zuo ZX, et al. Systematic Analysis of the Aberrances and Functional Implications of Ferroptosis in Cancer. iScience. Jul 24 2020;23(7):101302. doi:10.1016/j.isci.2020.101302

38. Lian $\mathrm{H}$, Han YP, Zhang YC, et al. Integrative analysis of gene expression and DNA methylation through one-class logistic regression machine learning identifies stemness features in medulloblastoma. Mol Oncol. Oct 2019;13(10):2227-2245. doi:10.1002/1878-0261.12557

39. Zhao M, Chen Z, Zheng Y, et al. Identification of cancer stem cell-related biomarkers in lung adenocarcinoma by stemness index and weighted correlation network analysis. J Cancer Res Clin 
Oncol. Jun 2020;146(6):1463-1472. doi:10.1007/s00432-020-03194-x

40. Geeleher P, Cox NJ, Huang RS. Clinical drug response can be predicted using baseline gene expression levels and in vitro drug sensitivity in cell lines. Genome Biol. Mar 3 2014;15(3):R47. doi:10.1186/gb-2014-15-3-r47

41. Sturm G, Finotello F, Petitprez F, et al. Comprehensive evaluation of transcriptome-based cell-type quantification methods for immuno-oncology. Bioinformatics. Jul 15 2019;35(14):i436-i445. doi:10.1093/bioinformatics/btz363

42. Finotello F, Mayer C, Plattner C, et al. Molecular and pharmacological modulators of the tumor immune contexture revealed by deconvolution of RNA-seq data. Genome Med. May 24 2019;11(1):34. doi:10.1186/s13073-019-0638-6

43. Becht E, Giraldo NA, Lacroix L, et al. Estimating the population abundance of tissue-infiltrating immune and stromal cell populations using gene expression. Genome Biol. Oct 20 2016;17(1):218. doi:10.1186/s13059-016-1070-5

44. Thorsson V, Gibbs DL, Brown SD, et al. The Immune Landscape of Cancer. Immunity. Apr 17 2018;48(4):812-830.e14. doi:10.1016/j.immuni.2018.03.023

45. Zheng $\mathrm{Y}$, Huang $\mathrm{Y}, \mathrm{Bi} \mathrm{G}$, et al. Multi-omics characterization and validation of MSI-related molecular features across multiple malignancies. Life Sci. Apr 1 2021;270:119081. doi:10.1016/j.Ifs.2021.119081

46. Yuan H, Yan M, Zhang G, et al. CancerSEA: a cancer single-cell state atlas. Nucleic Acids Res. Jan 8 2019;47(D1):D900-d908. doi:10.1093/nar/gky939

47. Hou GX, Liu P, Yang J, Wen S. Mining expression and prognosis of topoisomerase isoforms in nonsmall-cell lung cancer by using Oncomine and Kaplan-Meier plotter. PLoS One. 2017;12(3):e0174515. doi:10.1371/journal.pone.0174515

48. Bi G, Zhu D, Bian Y, et al. Knockdown of GTF2E2 inhibits the growth and progression of lung adenocarcinoma via RPS4X in vitro and in vivo. Cancer Cell Int. Mar 23 2021;21(1):181. doi:10.1186/s12935-021-01878-z

\section{Figures}


A

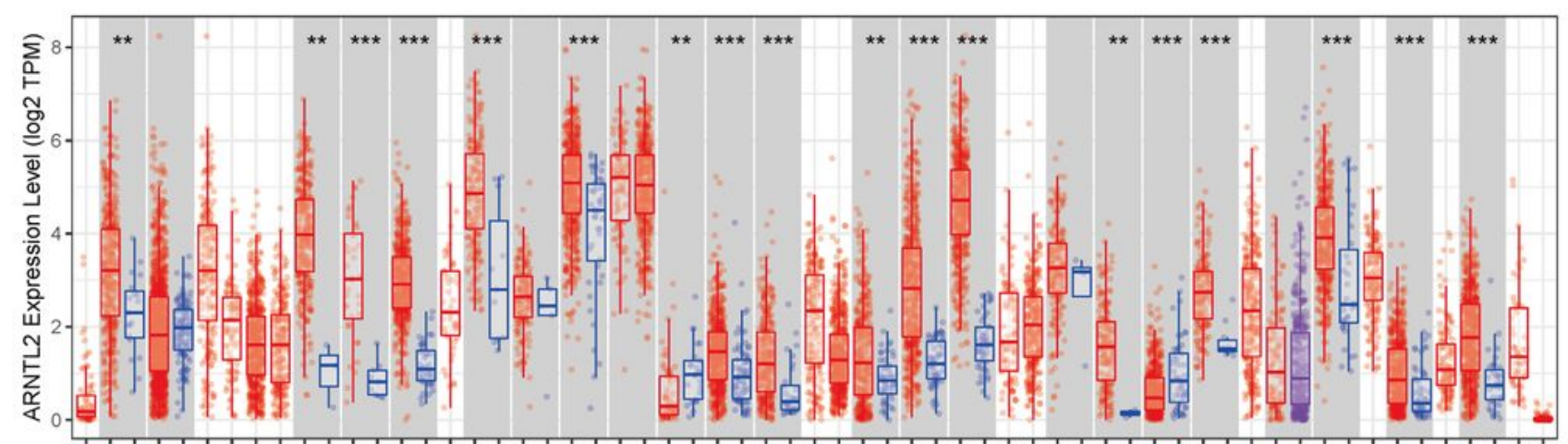

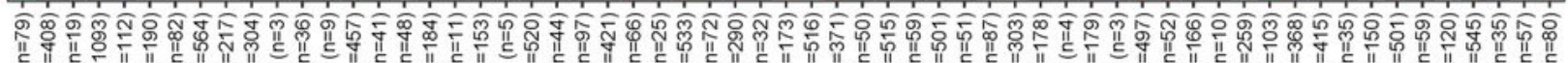

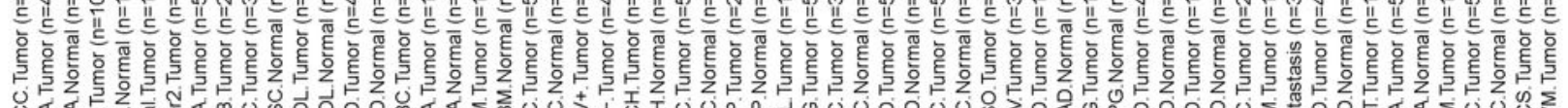

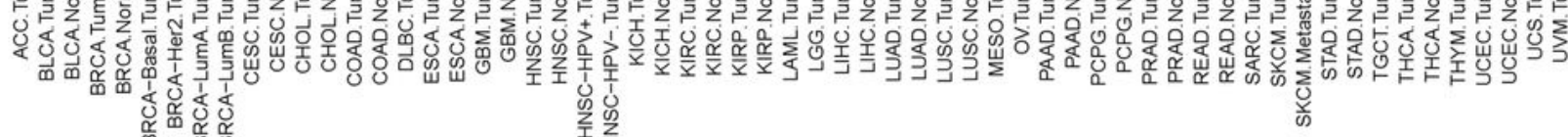

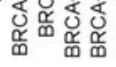

B

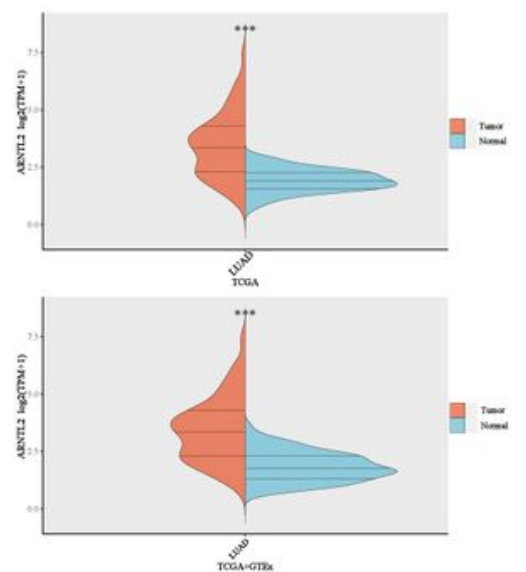

C

D
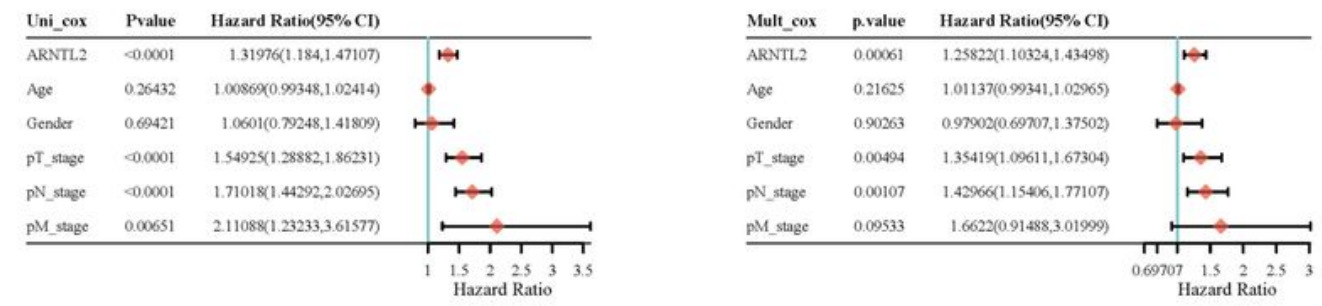

\section{Figure 1}

A ARNTL2 expression levels in different tumor types from TCGA database were determined by TIMER. B differences of ARNTL2 expression in lung adenocarcinoma tissues and normal tissues. C Survival curves comparing the high and low expression of ARNTL2 in lung adenocarcinoma. D Univariate and multivariate analysis of overall survival in LUAD patients from TCGA database. 
A

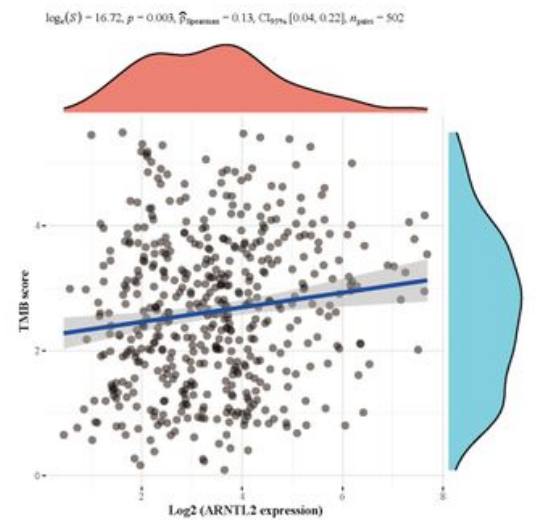

B

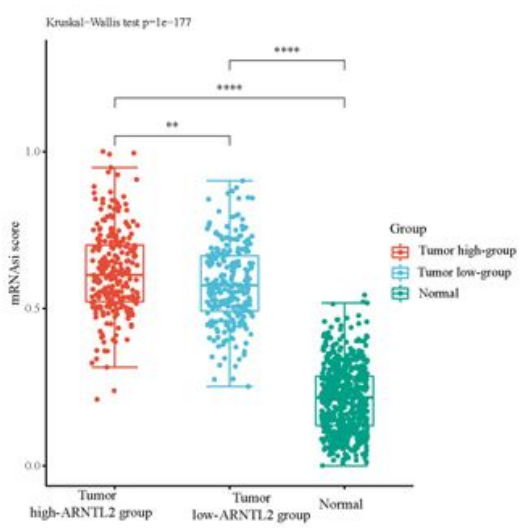

C

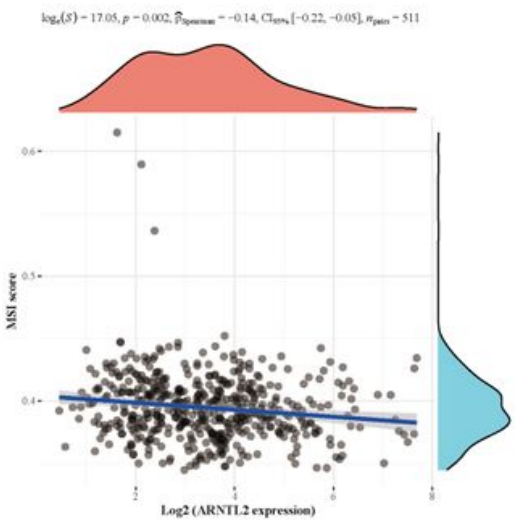

D
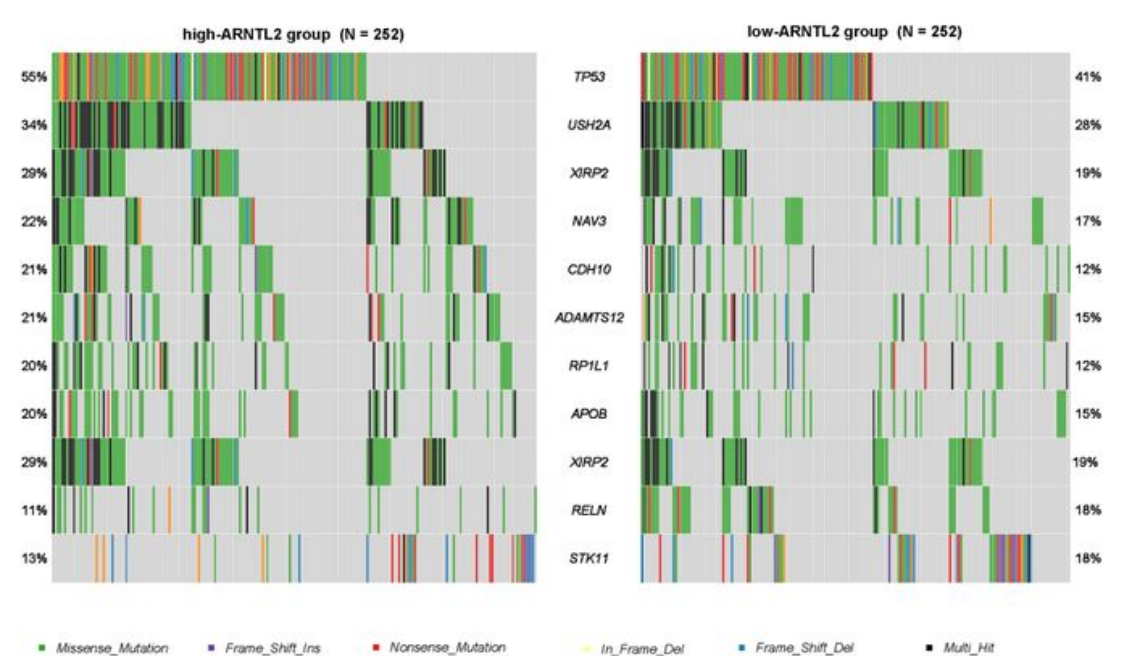

Figure 2

A Scatterplots of correlations between ARNTL2 expression and TMB. B The comparison of mRNAsi in high, low ARNTL2 expression groups of lung adenocarcinoma tissues versus normol tissues. C Scatterplots of correlations between ARNTL2 expression and MSI. D Differential mutations and their distributions in the high and low-ARNTL2 expression groups. 
A

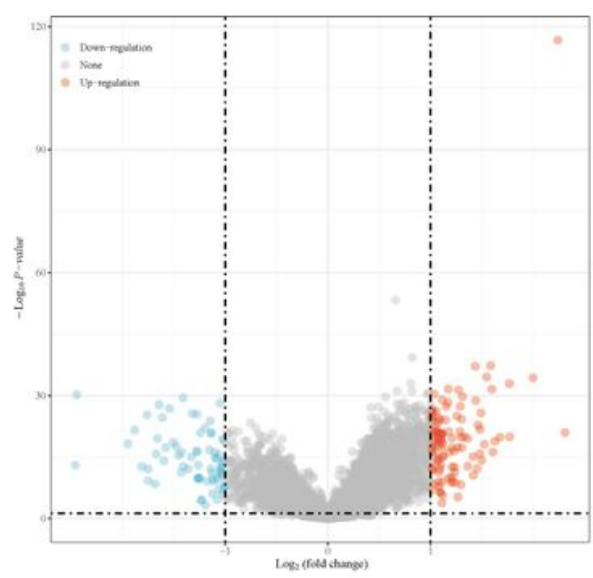

C

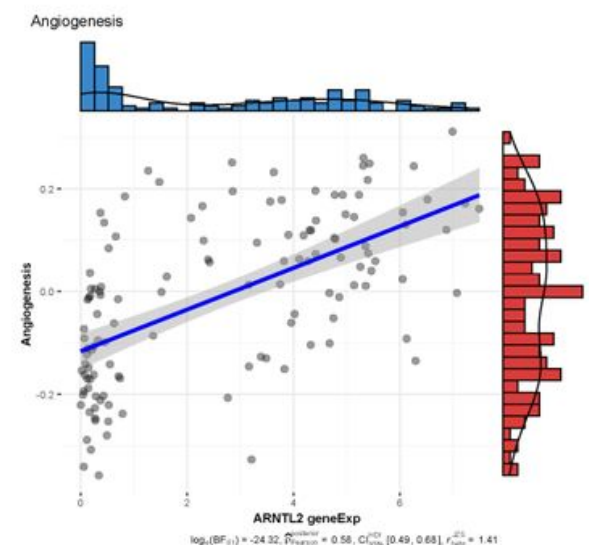

B
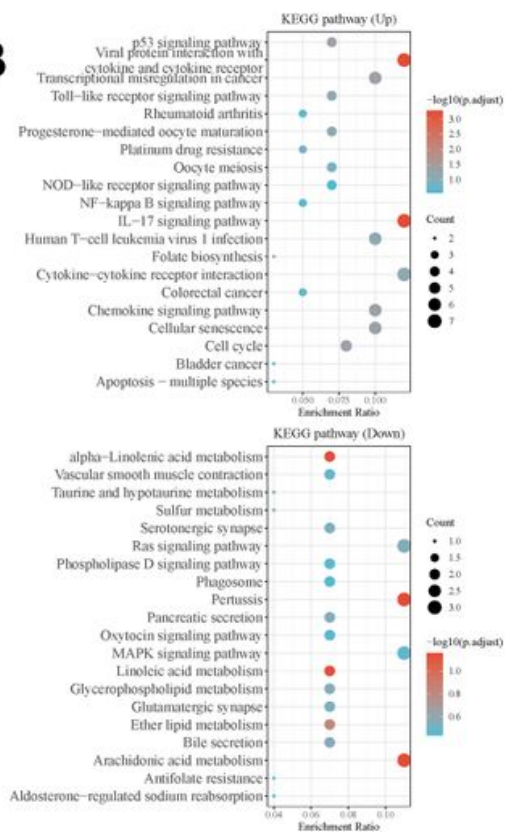

EMT
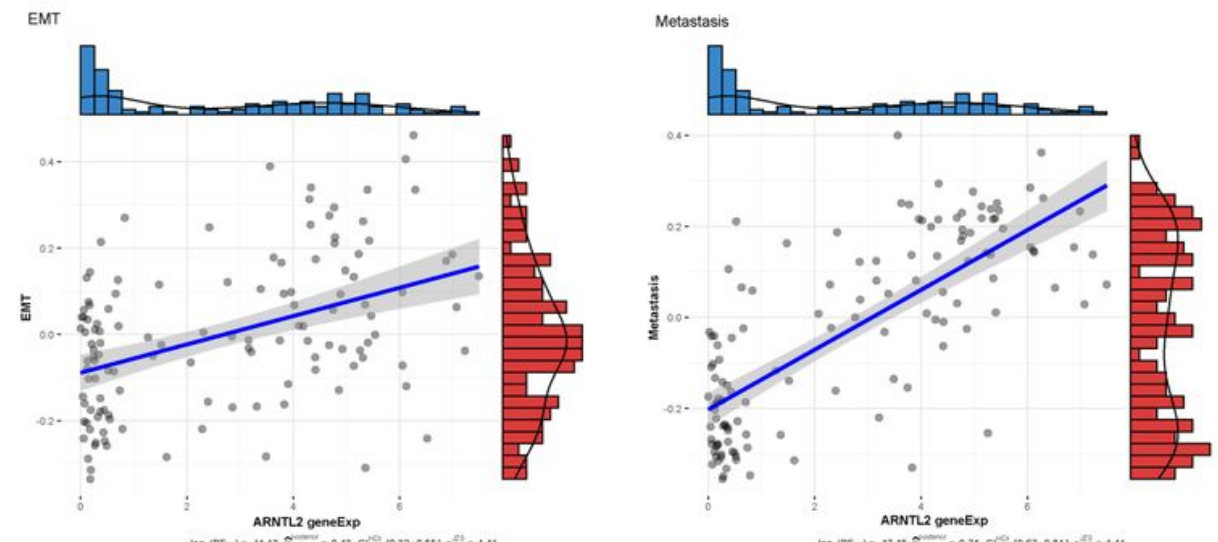

Figure 3

A Differential expressed genes between high and low-ARNTL2 expression groups were shown in a volcano plot. B Dot plot of GO and KEGG pathway analysis to the DEGs. C Scatterplots of correlations between ARNTL2 expression and angiogenesis, EMT and Metastasis by single-cell analysis of lung adenocarcinoma cells through the CancerSEA database. 

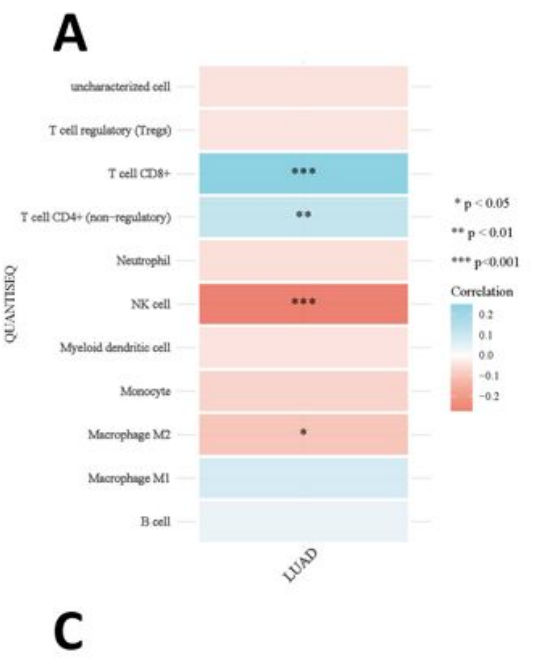

B

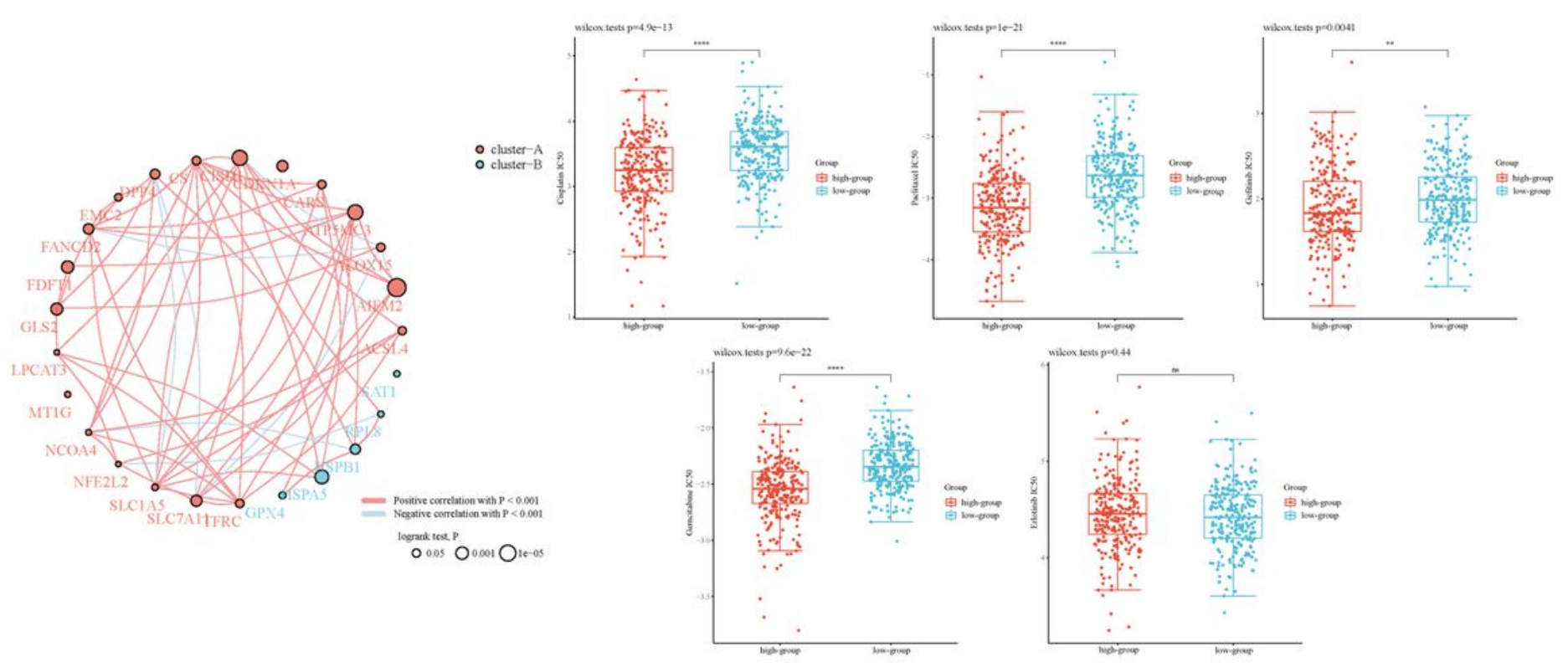

\section{Figure 4}

A Correlation of ARNTL2 expression with immune infiltration level in LUAD through QUANTISEQ method. $B$ Differences in the expression of some important immune gene closely related to immune checkpoints between high and low-ARNTL2 groups. C Differences in the expression of important genes associated with ferroptosis between high and low-ARNTL2 groups. D Relationship between drug sensitivity in lung adenocarcinoma and ANRTL2 expression were shown in box plot: Cisplatin, Paclitaxel, Gefitiinib, Gemcitabine and Erlotinib 
A

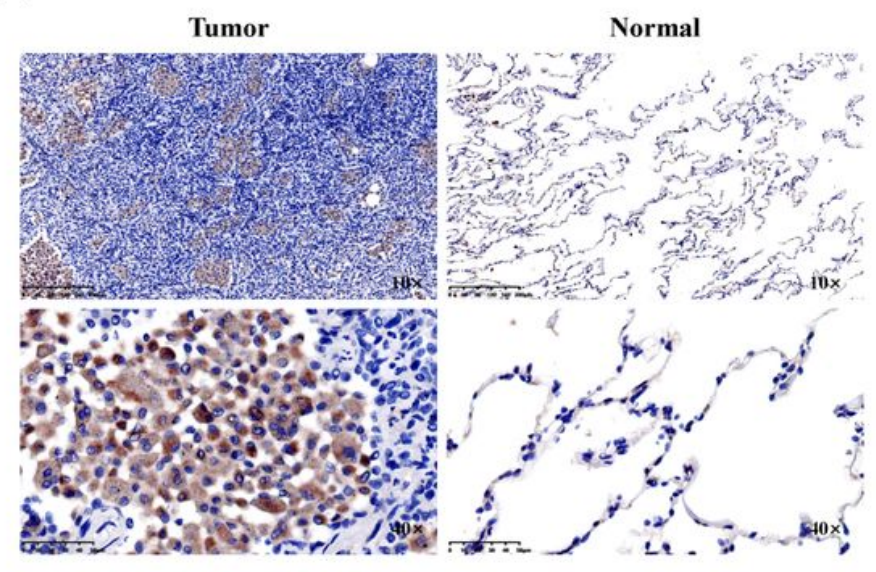

C

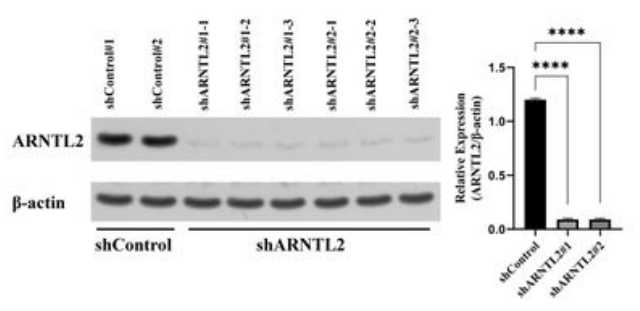

B
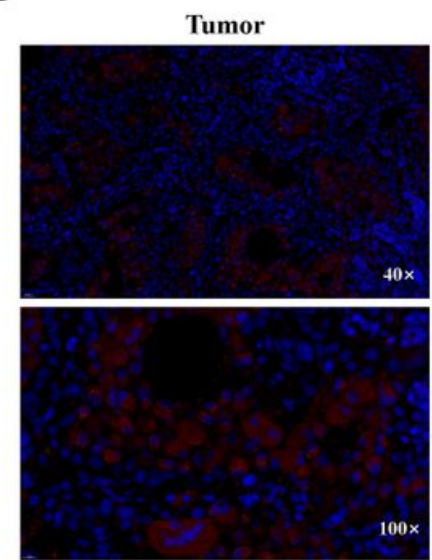

$40 x$

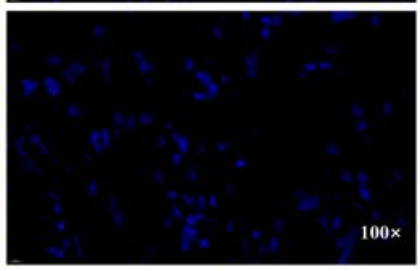

D

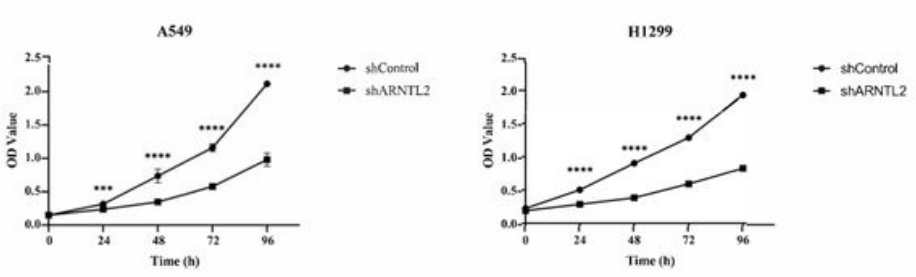

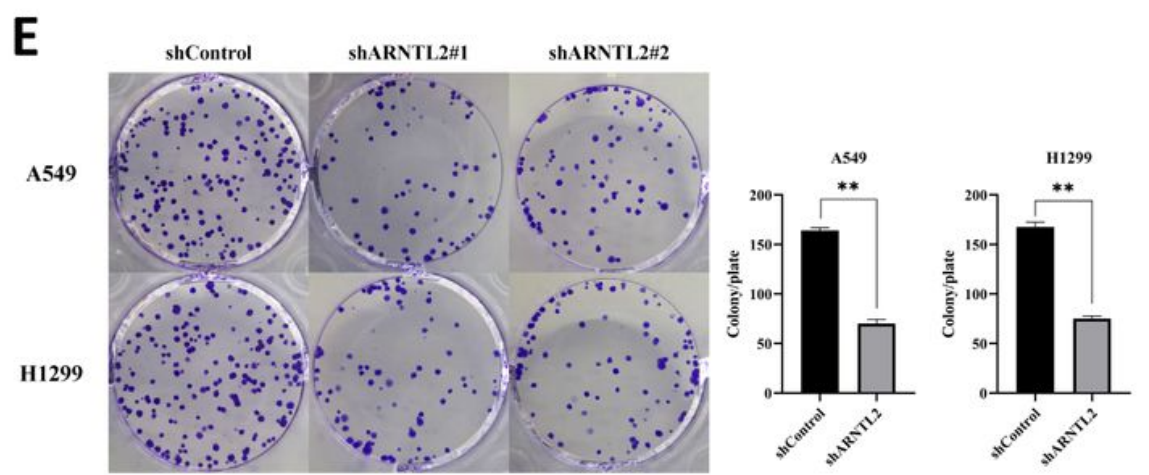

\section{Figure 5}

A-B The IHC (A) and immunofluorescence (B) staining of ARNTL2 in LUAD tissue versus adjacent normal tissues: ARNTL2 expression in tumor tissues was significantly higher than that in adjacent normal tissues. C Western blotting analyses verifying the ARNTL2 knockdown efficiency, quantification was performed by ImageJ: ARNTL2 knockdown was very efficient. D The effects of ARNTL2 knockdown on cell proliferation in A549 and H1299 cells through CCK8 assays: knockdown of ARNTL2 dramatically inhibited PDAC cells proliferation. E Comparison of colony formation efficiency between ARNTL2knockdown group and control group: knockdown of ARNTL2 dramatically inhibited colony formation of LUAD cells. 
A
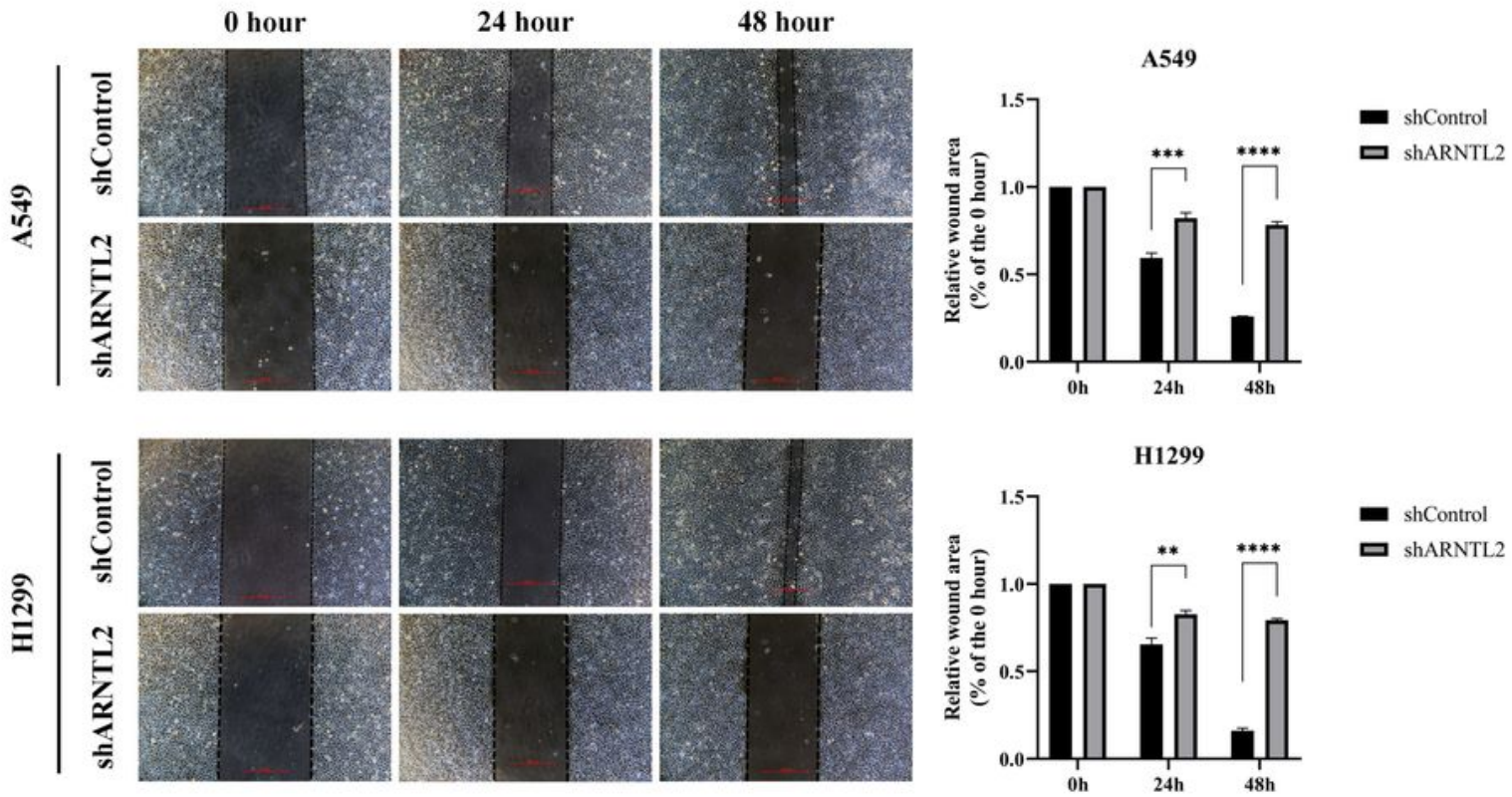

B

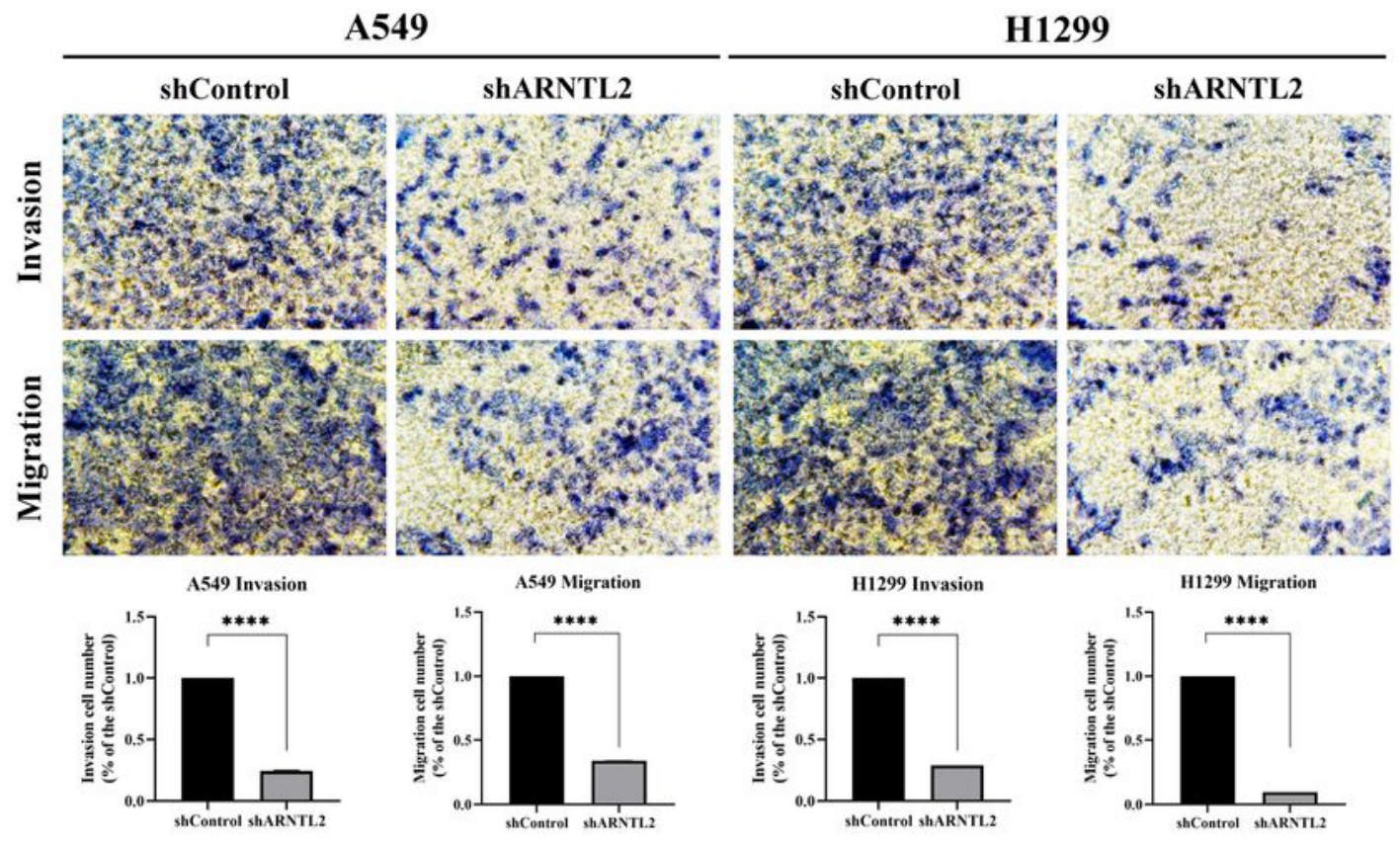

Figure 6

A Comparison of Wound-healing assays between ARNTL2-knockdown group and control group: ARNTL2 knockdown decreases cell migration in LUAD cells compared to control cells. B The invasion and migration capability of LUAD cells transfected with NC or sh-ARNTL2 was analyzed by transwell assay: ARNTL2 knockdown diminished migration and invasion ability of LUAD cells. 


\section{Supplementary Files}

This is a list of supplementary files associated with this preprint. Click to download.

- Figs1.tif

- FigS2.tiff

- TableS1shRNA.xIsx

- Tables2DEGs.csv

- TableS3PlotDatacorrheatmap.csv 